\title{
Discours
}

Revue de linguistique, psycholinguistique et

informatique. A journal of linguistics, psycholinguistics

and computational linguistics

$7 \mid 2010$

Varia

\section{Le couple interactif texte / phrase dans l'histoire de la langue française}

\section{Sabine Lehmann}

\section{OpenEdition}

Journals

Édition électronique

URL : http://journals.openedition.org/discours/7991

DOI : 10.4000/discours.7991

ISSN : 1963-1723

Éditeur :

Laboratoire LATTICE, Presses universitaires de Caen

\section{Référence électronique}

Sabine Lehmann, « Le couple interactif texte / phrase dans l'histoire de la langue française », Discours

[En ligne], 7 | 2010, mis en ligne le 20 décembre 2010, consulté le 19 avril 2019. URL : http:// journals.openedition.org/discours/7991 ; DOI : 10.4000/discours.7991

\section{(c) (i) (3) $\Theta$}

Discours est mis à disposition selon les termes de la licence Creative Commons Attribution - Pas d'Utilisation Commerciale - Pas de Modification 4.0 International. 

Revue de linguistique, psycholinguistique et informatique

\section{Le couple interactif texte/phrase dans l'histoire de la langue française}

Sabine Lehmann

MoDyCo, Université Paris Ouest, Nanterre La Défense

Sabine Lehmann, «Le couple interactif texte/phrase dans l'histoire de la langue française», Discours, 7| 2010, [En ligne], mis en ligne le 20 décembre 2010.

URL : http://discours.revues.org/7991. Consulté le 20 décembre 2010.

revues.org CENTRE POUR LÉDIITION ÉLECTRONIQUE OUVERTE
CENTRE FOR OPEN ELECTRONIC PUBLISHING

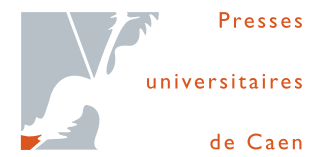

de Caen 



\title{
Le couple interactif texte/phrase dans I'histoire de la langue française
}

\author{
Sabine Lehmann \\ MoDyCo, Université Paris Ouest, Nanterre La Défense
}

\begin{abstract}
Cette étude est consacrée à la question de la cohérence textuelle dans différents types de textes en moyen français (discours scientifiques, ouvrages didactiques, récits brefs). Nous mettons au premier plan l'ensemble texte/phrase et montrons sous quelles conditions l'évolution du système linguistique produit de nouvelles structures textuelles. Nous décrivons les différents types de progressions thématiques caractérisant les productions textuelles en ancien et moyen français. La notion de genre est un critère important pour l'analyse de la macro- et microstructure des textes. Notre étude montre que l'évolution des structures textuelles est liée au niveau syntaxique et se manifeste dans la complexité de plus en plus grande des structures thématiques en moyen français. La relation qui s'établit entre le niveau thématique et les niveaux morphosyntaxique et sémantique est définie comme une interaction dont le fonctionnement est analysé dans une perspective diachronique.
\end{abstract}

Mots clés: moyen français, cohérence textuelle, genre, structures textuelles, microstructure, macrostructure, progression thématique

This study is devoted to the question of textual coherence in various types of texts in Middle French (didactic works, short stories). The goal is to highlight the text/sentence (global set) assembly and to show under which conditions the evolution of the linguistic system produces new textual structures. We describe here the different types of theme progression used in the textual productions in Middle French. The concept of genre is an important parameter for the analysis of macro-and microstructure of a text. Our study shows that the textual structures evolution is related to the syntactic level and appears in the increasing complexity of thematic structure sets in Middle French. The relation which is established between the thematic level and the morphosyntactic and semantic levels is defined as an interaction whose operation is analyzed in a diachronic perspective.

Keywords: Middle French, textual coherence, genre, textual structures, microstructure, macrostructure, theme progression

\section{Introduction}

Dans le cadre de notre étude, nous nous intéressons au couple interactif texte/phrase dans l'histoire de la langue française. Après avoir caractérisé l'évolution des séquences textuelles en ancien français, nous montrerons ce que le texte fait à la phrase, pendant la période du moyen français $\left(\mathrm{XIV}^{\mathrm{e}}-\mathrm{XV}^{\mathrm{e}}\right.$ siècles). Notre travail s'inscrit dans le cadre des recherches effectuées à l'occasion du colloque international «Le texte: modèles, méthodes, perspectives» (25-27 septembre 2008, Cluj, Roumanie) et centrées sur la question de la cohérence textuelle en ancien et moyen français. Nous nous interrogerons sur la nécessité de la prise en considération de l'ensemble texte/phrase et sur la nature de l'interaction qui s'établit entre ces deux unités. Il nous semble important de relativiser la question de la phrase comme unité de 
base de la textualité. Le texte en tant que «suite signifiante (jugée cohérente) de signes entre deux interruptions marquées de la communication» (Weinrich, 1973: I3 et 198), possède la particularité de constituer une totalité dans laquelle des éléments de niveaux différents de complexité entretiennent les uns par rapport aux autres des relations d'interdépendance. Nous proposons de considérer la phrase comme un palier d'organisation situé entre signes et propositions, d'une part, et paragraphes, séquences et parties d'un plan de texte, d'autre part. La phrase en tant qu'élément constitutif de micro- et macropropositions entre dans la composition de superstructures séquentielles dont la disposition représente l'aspect compositionnel d'un texte. Un plan de texte conventionnel est fixé par le genre de discours. Nous nous appuierons sur cette notion de genre pour sortir de l'impasse théorique résultant d'une conception du texte comme addition de séquences.

Au sein du discours, les phrases entretiennent des liens de cohésion qui contribuent à ce que M.A.K. Halliday et R. Hasan (1976) appellent sa «texture». La mise en place de cette texture se manifeste à travers des phénomènes qui dépassent le niveau de la phrase comme, par exemple, les différents types de progressions thématiques, les enchaînements de phrases ou la présentation des éléments nouveaux. Il s'agit de montrer dans quelle mesure le système linguistique permet, par son évolution, l'apparition de nouvelles structures textuelles.

Le niveau d'organisation, de structuration d'un ensemble textuel est particulièrement intéressant dans la mesure où il constitue un paramètre d'analyse aussi bien pour l'objet complexe texte - et donc aussi pour le discours, si l'on décide de relier texte et discours dans un rapport de "profonde complémentarité», comme le reconnait Adam ${ }^{1}-$, que pour la phrase, dont le degré de dynamisme communicatif et la structure syntaxique dépendent de sa place à l'intérieur des différentes séquences textuelles qui forment l'objet complexe texte. Quant au mode de formation de la totalité textuelle, c'est l'application du concept de plan de texte qui nous permettra de comprendre l'organisation globale d'un ensemble textuel et d'ouvrir la voie à un autre paramètre important pour la conceptualisation de la totalité textuelle: la notion de genre. Ainsi, comme l'a constaté J.-J. Richer (2004: I25):

Par le biais du concept de plan de texte, la notion de genre trouve place dans la conceptualisation de la textualité de J.-M. Adam où, dans le prolongement de la définition avancée par D. Maingueneau, le genre se voit doté de six caractéristiques [ou niveaux] (1997: 5): sémantique (thématique), énonciatif, pragmatique, compositionnel, longueur, stylistique.

La prise en considération du concept de genre nous semble particulièrement pertinente dans une étude qui s'attache à la diachronie du français et surtout à la période du moyen français. En effet, cette période est caractérisée par le développement du

1. «Le présent ouvrage plaide ainsi, non pour la confusion des domaines, mais, lors de l'analyse, pour la démonstration de leur profonde complémentarité» (Adam, I999: 4I). 
texte argumentatif et, plus largement, du texte informatif et explicatif, rédigé en langue française. Les œuvres scientifiques, philosophiques et politiques sont de plus en plus rédigées en français, ce qui entraîne la mise en place de nouveaux types de progressions thématiques et des contraintes nouvelles concernant la disposition textuelle. Nous développerons davantage l'analyse de la relation qui s'établit entre le genre et les contraintes de disposition dans la partie de notre étude consacrée à la macrostructure des textes de notre corpus. Quant aux textes choisis pour notre étude, nous accorderons une importance particulière aux textes représentatifs du genre argumentatif et explicatif, en l'occurrence le discours scientifique (la traduction française du XV ${ }^{\mathrm{e}}$ siècle de la Chirurgia Magna de Guy de Chauliac; les traductions françaises du Compendium de epidemia) et le discours didactique (Le Mesnagier de Paris). Le genre narratif jouera également un rôle, car le paysage littéraire aux $\mathrm{XIV}^{\mathrm{e}}-\mathrm{XV}^{\mathrm{e}}$ siècles s'enrichit et se diversifie grâce à l'évolution du genre narratif bref (nouvelles). Nous nous intéresserons surtout aux nouvelles qui sont regroupées dans des recueils (Les Cent Nouvelles Nouvelles; Les Quinze Joyes de mariage; L'Heptaméron) et pour lesquelles les notions de disposition textuelle ainsi que de macro- et microstructure textuelle constituent donc des paramètres d'analyse intéressants.

\section{Le couple texte/phrase et la notion de progression thématique en diachronie}

Les notions de texte/discours, phrase et genre correspondent à des catégories de description nécessaires pour rendre compte des textes en tant qu'objets empiriques et donc, complexes ${ }^{2}$. Le texte, à travers sa tonalité générale, la stratégie discursive qui y est développée - en bref - de par son appartenance à un genre (qu'il reproduit ou innove), impose aux séquences qui le constituent un certain type d'organisation se traduisant dans les enchaînements de phrases, le regroupement de séquences qui forment des sous-ensembles textuels, ainsi que dans le choix de différents types de progressions thématiques.

Le plan thématique d'un énoncé peut être considéré «à l'intérieur» de la phrase (découpage en unités, correspondance avec des positions syntaxiques), mais doit aussi être analysé en tant que facteur de cohérence: nous retrouvons là la question des divers types de progressions thématiques et le rôle de la perspective fonctionnelle dans les enchaînements de phrases ${ }^{3}$. Les éléments qui contribuent à la cohésion

2. Nous alignons notre réflexion sur les analyses faites par Antónia Coutinho (2004:32-33) concernant la complémentarité entre discours et texte: «Je prendrai donc discours, genre et texte comme des “catégories descriptives" nécessaires pour rendre compte des objets complexes que sont les textes empiriques. [...] Au discours, envisagé comme objet de dire, j’opposerai le texte, comme "objet de figure" - en reprenant et réorganisant ainsi des notions proches qui apparaissent, sous différentes formulations, dans des cadres théoriques distincts (quoique proches, comme ceux de la logique naturelle et de la linguistique textuelle) et éloignés dans le temps, comme dans le cas de l'héritage de la rhétorique».

3. Selon la ligne tracée par l'École de Prague, l'objectif principal affiché par Mathesius (I964) et à sa suite, par Daneš (1974) et Firbas (I97I et 1974), par exemple, était bien d'appréhender l'articulation entre le 
textuelle ne peuvent toutefois être dissociés des facteurs qui régissent la structure interne de la phrase. C'est cette mise en relation entre le texte et la phrase qui nous intéresse dans le cadre de notre étude.

Les analyses des mécanismes de l'organisation du texte, qui permettent d'en distinguer les unités structurelles tout en assurant sa cohésion et la continuité thématique et référentielle, sont maintenant bien représentées dans les travaux sur l'évolution de la langue française (par exemple Combettes, 1986, I999 et 2007; Bazin-Tacchella, 2005 et 2007). C'est surtout avec les travaux de Vennemann (I973, 1974) que la notion de thème s'introduit peu à peu dans le champ d'étude de la syntaxe diachronique. Comme l'a souligné B. Combettes (1999: 232), l'utilisation de cette notion ne s'accompagne pas toujours d'une grande clarté dans les définitions ou même dans la terminologie. Selon une première approche, le thème devient le «point de départ» de l'énoncé à partir duquel une description/argumentation sera développée, ou qui va servir de cadre à la suite de la phrase. L'autre grande direction est constituée par les essais visant à caractériser le thème en prenant en compte la charge informative des divers constituants, et ceci grâce à l'établissement d'une hiérarchie au niveau du dynamisme communicatif. Dans cette perspective, le thème serait alors l'élément de plus bas degré dans la hiérarchie du dynamisme communicatif. Afin d'éviter une polysémie quelque peu gênante, B. Combettes propose de «réserver des notions telles que celles de thème et de rhème pour l'analyse de ce niveau informationnel, dans la ligne de la Perspective Fonctionnelle de la Phrase (FSP), alors que l'articulation de l'énoncé en "point de départ" et en développement de la prédication pourrait être décrite par des termes comme ceux de topique et de commentaire» (1999: 232).

Il faut toutefois remarquer que le niveau informationnel ou thématique est rarement étudié en tant que tel, mais que son analyse est mise à contribution pour rendre compte de faits qui relèvent habituellement de la syntaxe et qui semblent exiger une approche contextuelle dépassant le cadre de la phrase. Une telle utilisation subordonne en quelque sorte l'étude du niveau informatif à celle de la structuration syntaxique de l'énoncé. Cette emprise de la syntaxe sur la perspective fonctionnelle de la phrase a peut-être, dans un premier temps, empêché une réflexion plus approfondie sur la spécificité de l'organisation en thème/rhème, sur la nature propre de ce domaine et sur son application aux études diachroniques. Des travaux plus récents adoptant une approche diachronique (B. Combettes, C. Buridant, S. Bazin-Tacchella, S. Lehmann) témoignent d'un élargissement de la problématique en vue d'un nouvel équilibrage entre le niveau thématique et le niveau syntaxique. Toutefois, cette nécessité d'élargissement ne remet pas en question l'idée centrale de notre étude selon laquelle l'examen du domaine thématique met en jeu deux différents types d'unités: la structure

niveau thématique et le niveau syntaxique, ou, plus précisément, d'examiner comment les formes, les constructions, assurent le codage de la hiérarchie des degrés du dynamisme communicatif. Pour ce point, voir aussi les travaux de P. Sgall (I974) et E. Hajičova (1994). 
propositionnelle, où doit s'organiser la linéarisation des divers syntagmes, et le niveau textuel, où s'enchaînent les propositions successives; les fonctionnements respectifs de ces deux unités, en ce qui concerne le plan informationnel, étant étroitement reliés.

La thématisation, opération qui produit des effets sur l'organisation du discours, s'observe à travers la succession et la transformation des objets de discours. Ces derniers se déploient dans la durée discursive; ils sont travaillés, transformés et s'observent par le biais de leur circulation dans le discours. D'un point de vue diachronique, l'organisation du discours et la gestion des objets de discours obéissent à des principes de plus en plus complexes.

Les textes d'ancien français qui appartiennent essentiellement au type narratif représentent une conception assez resserrée de la cohérence textuelle selon le modèle: Thème + Verbe + «Reste», la charge informative de cette deuxième partie étant très diverse. Très souvent, ce sont des marqueurs de cohérence comme si et or qui rattachent chaque phrase à celle qui la précède. Les éléments nouveaux ne peuvent être introduits qu'après un tel ancrage dans le contexte, le verbe apparaissant comme un élément intermédiaire, souvent introducteur de ces groupes rhématiques. Dans son étude consacrée à l'Évolution des structures thématiques en moyen français, B. Combettes (2007:36) a montré que ces groupes rhématiques sont relativement hétérogènes sur le plan informationnel:

Une conséquence directe de cette dichotomie est la grande diversité, du point de vue du dynamisme communicatif, des constituants qui se trouvent placés dans la zone postverbale. Si l'on considère par exemple le cas du sujet «inversé», on pourra constater que ce syntagme peut renvoyer non seulement à un référent nouveau, ce qui est attendu dans la partie rhématique de la proposition, comme dans: $A$ ceste parole leva la noise el palais ( $L$ a Mort Artu, XIII ${ }^{e}$.), mais également à un référent identifiable qu'il est nécessaire de réactiver, dans la mesure où il n'a pas été mentionné dans le contexte proche: A cest conseil survint Hector (ibid.).

Selon le système de l'ancien français, l'importance première est donc accordée à l'identification du thème. Le schéma de phrase à verbe second avec sujet «inversé» $(\mathrm{V}+\mathrm{S}+\mathrm{X})$ produit des séquences figées avec une zone postverbale dont les diverses composantes rhématiques ne sont pas marquées en ce qui concerne leur degré de dynamisme communicatif.

La syntaxe à verbe second se voit modifiée, relativement tôt, par l'apparition de structures comportant deux constituants avant l'élément verbal. Très souvent, il s'agit de circonstants de lieu ou de temps qui portent le même degré de dynamisme communicatif et se combinent dans la zone préverbale:

[r] Au soir après souper avint que li sires de l'ostel s'ala esbatre en un prael qui estoit derriers sa meson.

(La Mort le roi Artu, § 26, 1. I-3) 
On doit signaler, dans ces faits de double thématisation, l'importance des propositions subordonnées circonstancielles. En effet, la combinaison d'un circonstant temporel et d'une subordonnée temporelle est bien attestée en position thématique:

[2] L'endemain, quant il fu jorz, vindrent a un chastel ou li rois avoit jeü la nuit [...].

(La Mort le roi Artu, § Iо, 1. I-2)

Les productions littéraires en ancien français appartiennent, comme nous l'avons déjà souligné, essentiellement au genre narratif. Le type d'écriture pratiqué par les auteurs de cette période est caractérisé par des liaisons interphrastiques à courte distance et privilégie la progression à thème constant et la progression linéaire. Comme nous l'avons montré dans notre étude réalisée à l'occasion du colloque international «Le texte: modèles, méthodes, perspectives» (Lehmann, 20ıо : 149), dans ce type d'enchainement, constant dans les textes du XIII siècle, l'introduction des syntagmes rhématiques se fait essentiellement par l'intermédiaire de verbes de perception et d'action:

[3] Si regarde contremont et voit venir une main toute enflamee qui le feri [...] Lors oï une voiz qui li dist [...].

(La Queste del Saint Graal (1220), § 253, 1. 20)

[4] Lors s'afiche Lancelos seur les estriés et se met enmi les rens et fiert un chevalier qu'il encontra [...]. Il hurte outre por parfere son poindre, car ses glaives n'estoit pas encore brisiez; si ataint un autre chevalier et le fiert si que [...] Il l'empaint bien, si le porte jus del cheval a terre [...].

(La Mort le roi Artu, § 18, 1. I-4)

Dans son étude consacrée à L'articulation des phrases narratives dans «La Mort Artu», J. Rychner a étudié ce dernier exemple en insistant sur le fait que «le rapport entre la phrase à sujet nominal et la phrase en $i l$ n'est de thème à prédicat que dans la faible mesure où un procès narratif est toujours prédicat du procès précédent» (1970: 56). On assiste à un phénomène de distribution de différents degrés de dynamisme communicatif liés aux procès désignés par les verbes. En effet, il est intéressant de constater que les procès en il (il hurte, il l'empaint) sont présentés comme plus libres et nouveaux vis-à-vis des procès précédents que les procès en si (si ataint, si le porte) ne le sont vis-à-vis des procès en il. Ces procès à différents degrés de dynamisme communicatif sont associés à un sujet nominal, introducteur de la série d'actions, et ensuite à un sujet pronominal qui «entame donc l'étape dans laquelle se range la particule» (Rychner, 1970: 57). Le poids informatif des procès se détermine donc en fonction de la nature du sujet ainsi que de l'éloignement des sujets pronominaux par rapport à leur antécédent.

Comme nous pouvons le constater dans les exemples [3] et [4], c'est le recours à la vision ${ }^{4}$ ou aux actions d'un personnage qui sert de relais pour l'insertion de groupes

4. Les verbes de perception introduisent «un sujet nouveau, dans la perception duquel personnage, objet ou situation antérieurement désignés seront ainsi transférés : la reine prist un message [...] Et quant messire 
indéfinis à valeur rhématique dans la narration. La cohérence textuelle est établie par l'utilisation d'un sujet-thème identique (qui n'est pas toujours exprimé) dont les aspects différents (composantes rhématiques: Rhème I, Rhème 2, Rhème 3...) sont dévoilés dans une perspective chronologique qui suit les diverses perceptions ou actions du ou des personnage(s). Un circonstant thématique (lors) ou la "particule» $s i^{5}$ renforcent la cohérence déjà établie par l'utilisation d'un sujet identique. Ce type de progression thématique produit des fragments de texte à valeur descriptive qui n'apparaissent pas comme autonomes, car «la description, si développée soit-elle, ne fait pas l'objet d'un énoncé indépendant, mais elle est insérée dans la narration, les éléments descriptifs dépendant du verbe introducteur» (Combettes et Tomassone, 1985: 78).

La progression à thème linéaire, l'autre grand procédé attesté, fonctionne selon un principe de transformation: chacune des phrases débute par un thème qui reprend le rhème ou une partie du rhème de la phrase qui précède. Le recours à la vision d'un personnage n'est plus indispensable; ce type de progression entraîne plutôt la présence de tours impersonnels, comme dans cet extrait de La Suite du roman de Merlin (1235):

[5] Quant il vint a demie liue priés du chastiel, il trouva une grant chimentiere ou il avoit tombes plusiors vielles et nueves. Au chief del chimentiere par devers le chastiel avoit une crois toute nueve. En cele crois avoit letres qui disoient [...].

$$
(\S 225,1 . \mathrm{I}-5)
$$

Ces deux types de progression - à thème linéaire et à thème constant - répondent donc bien au modèle: Thème + Verbe + «Reste». On les retrouve encore en moyen français, dans les ouvrages qui maintiennent le «vieux style» ${ }^{6}$, caractérisé par des enchaînements qui se font d'une phrase sur l'autre, sans rupture. Nous sommes en face de séquences «en escalier» ou «en cascade» à reprise thématique: le rhème

Lancelos vit le messaje» (La Mort le roi Artu, § 89, 1. I; Rychner, 1970: 108).

5. J. Rychner a montré que lors marque généralement dans le texte un début plus important que si. Cet adverbe «occupe bien plus souvent que si une place faite pour l'attaque, et bien moins souvent une position où la phrase précédente pourrait se continuer» (I970: 156).

6. Dans leur article centré sur les progressions thématiques, B. Combettes et R. Tomassone (1985: 79) constatent que cette façon d'assurer la cohérence textuelle «n'est pas réservée aux textes de "fiction", aux romans de chevalerie; chroniqueurs, historiens, adoptent la même présentation: des passages entiers, chez Froissart, sont articulés autour des personnages:

- Et tantost après mienuit, il sallirent sus, sans faire grant noise, et s'ordennerent en pluisseurs routes, et s'en vinrent pour assallir Vennes. Et fissent alumer grant fuisson de feus de busce [...] Chil qui faisoient le gait pour la nuit dedens Vennes veirent les feus eslever contremont a celle heure la. Si furent tout esmervilliet [...] si vinrent celle part; si conmenchierent a faire grant noise et a resvillier ceuls qui dormoient (571).

- Et coururent chil de l'avant-garde jusques a Aumale et fu la ville arse; et puis s'en retournerent viers Oizemont [...] Et trouverent sur lor chemin une grosse ville non fremee qi s'appelle Senartpont [...] ; et puis passerent oultre et vinrent a Oizemont. La trouverent il le roi et toute l'oost; si se logierent pour ce jour (705)». 
d'une première phrase y devient le thème d'une seconde phrase ${ }^{7}$. L'articulation des séquences textuelles se fait donc par des phénomènes de reprise qui saturent le récit et refusent le vide. Comme l'ont montré B. Cerquiglini, J. Foret et D. Mukherjee (1976: 45-64), la transition entre deux actions peut être réalisée sous forme de propositions temporelles, qui marquent des étapes tout en fonctionnant selon le mode de la reprise ${ }^{8}$. Le style narratif des chroniqueurs des XIII ${ }^{e}-\mathrm{XIV}^{\mathrm{e}}$ siècles répond à cette étiquette de «vieux style» qui privilégie des enchânements rectilignes par installation de relais et une décomposition de l'action.

En revanche, le développement et l'essor du texte argumentatif pendant la période du moyen français vont de pair avec une autre conception de la cohérence discursive qui s'écarte nettement des liaisons interphrastiques évoquées plus haut. En effet, la cohérence est assurée par l'établissement d'un hyperthème, c'est-àdire par le renvoi à une unité supérieure qui sert de point de référence. Comme nous l'avons montré dans notre article consacré à la cohérence textuelle et aux progressions thématiques en moyen français (Lehmann, 20IO), le passage à ce type d'enchaînement plus global, qui se manifeste au niveau du discours, par exemple sous forme d'un terme collectif qui sera «subdivisé» en ses diverses parties, va de pair avec l'évolution d'un paysage littéraire privilégiant de plus en plus des textes descriptifs ou explicatifs qui peuvent se passer de l'intervention d'un personnage dont la perception ou les actions servent de relais à l'introduction de passages descriptifs. Au contraire, le fonctionnement par hyperthème équivaut à séparer plus nettement description et narration pour faire de la description un système à part pouvant fonctionner sans référence au domaine narratif. C'est en effet dans le type narratif, avec la prédominance des séquences renvoyant à une succession d'événements, que vont dominer la progression à thème constant et la progression linéaire. Dans la progression à thème constant, les fragments descriptifs sont inséparables de la narration et dépendent, comme nous l'avons souligné, de la vision d'un personnage. Les hyperthèmes - caractéristiques des textes argumentatifs en moyen français - sont des enchaînements à caractère global qui s'adaptent mieux à des textes descriptifs ou à des passages de commentaire, d'arrière-plan où la description s'énonce d'elle-même, sans l'intermédiaire d'une vision et sans enchaînement chronologique.

L'importance de la période du moyen français concernant l'écriture de textes non narratifs a été soulignée à plusieurs reprises. La prise en considération de ces nouveaux types de texte à caractère argumentatif/explicatif/informatif témoigne d'une nouvelle conception de la cohérence discursive et permet de constater dans quelle mesure le fonctionnement par hyperthèmes contribue à la complexification

7. Voir Buridant, 2000: 6II (§ 506$)$.

8. «Notre classe d'articulations (en et, puis en $e t+q u a n t)$ doit s'élargir, pour intégrer d'autres procédés, généralement anaphoriques (et + quant + reprise). Ainsi : Et il regardent les lettres... Et quant il voient ces lettres, si dient li uns a l'autre» (La Queste del Saint Graal, § 4, 1. 8; cité par Cerquiglini, Foret et Mukherjee, $1976: 5 \mathrm{I})$. 
des structures thématiques. Dans la troisième partie de notre étude consacrée à l'analyse des niveaux macro- et microstructurel des textes choisis, nous insisterons sur le rôle des hyperthèmes dans la construction du texte.

\section{De la macro- à la microstructure}

Cette partie de notre travail est centrée sur les objets empiriques, c'est-à-dire les textes de notre corpus qui seront étudiés du point de vue de leur organisation globale (macrostructure) et du point de vue de l'articulation des séquences textuelles grâce à des structurants textuels.

\subsection{La macrostructure: genre et disposition textuelle}

Comme nous l'avons déjà annoncé dans l'introduction, c'est donc par le biais du concept de "plan de texte», pour reprendre la formulation de J.-J. Richer, que la notion de genre trouve place dans la conceptualisation de la textualité de J.-M. Adam. En effet, ces plans de texte sont liés aux genres de discours: «Les plans de texte sont généralement fixés par l'état historique d'un genre ou d'un sous-genre de discours» (Adam, 1997: 5). La notion de genre doit, nous semble-t-il, être articulée avec celle de configuration et de disposition (textuelle). Même si la notion de configuration intègre le cadre de la logique naturelle, elle a été explicitement rapprochée de la problématique textuelle (J.-B. Grize, cité par Coutinho, 2004:34):

Nous appellerons configurations les diverses dispositions des énoncés les uns par rapport aux autres. Ce concept relève de ce que Halliday nomme «texture», c'està-dire le tissu même du texte.

Dans le cadre des études linguistiques sur le texte, c'est l'idée d'«orientation configurationnelle» proposée par J.-M. Adam, qu'il convient de rappeler. Dans son article intitulé «Schématisation (discursive) et disposition (textuelle)», A. Coutinho propose de réunir dans la notion de texte comme objet de figure (2004: 35) ces différents éléments: orientation configurationnelle, configuration, séquence, figure ${ }^{9}$, qui constituent l'unité du texte. Ce qui nous semble pertinent dans l'approche de cet auteur est le fait de considérer le genre comme catégorie reliant un texte à un type de discours. L'unité du texte se constitue d'après ce qui est prévu par le genre dans lequel il s'inscrit. Parmi les composantes du genre, il faut certainement évoquer la notion de compositionnalité qui implique l'identification des spécificités de l'agencement des textes du point de vue de leurs schémas d'organisation ou

9. Reprenant à Ricour la notion de configuration, Adam fait dépendre l'effet de texte du passage de la séquence à la figure, c'est-à-dire de la suite de propositions (ou dimension séquentielle), de type linéaire, à l'orientation configurationnelle (ou configuration pragmatique), d'ordre global (voir Adam, I990 et I992). 
superstructures ${ }^{10}$. L'importance de cet aspect organisationnel d'un ensemble textuel se traduit aussi à travers la notion de plan de texte que nous avons déjà évoquée comme étant une possibilité pour sortir de l'impasse théorique résultant d'une conception du texte comme addition de séquences et pour élaborer une autre voie de conceptualisation de la totalité textuelle.

Nous partons de l'idée que chaque genre prévoit - en tant que «moule» préétabli un certain type d'organisation de nature compositionnelle: «On pourra donc dire que le genre pré-figure le texte, ou que le genre définit ce qui, dans le texte empirique, fait figure - de texte» (Coutinho, 2004: 37). Dans le texte s'entrelacent différents axes organisationnels qui - au niveau de la structure globale du texte - correspondent aux plans de texte et qui reflètent les contraintes plus ou moins fixes ou réglées, imposées par le genre. Comme l'a souligné J.-J. Richer, la notion de plan de texte reste à définir pour éviter de n'être utilisée «qu'en un sens faible, ou non technique» (selon l'expression de Bronckart, 1997: 252) et "pour dépasser le stade d'une simple énumération de plans de textes plus programmatique que définitoire» (Richer, 2004: I26), comme le fait J.-M. Adam "1. Soulignons, tout en nous inspirant des recherches de J.-J. Richer, que la notion de plan de texte doit être éprouvée par un travail systématique genre par genre, car les plans de texte sont liés aux genres des discours. Enfin, la notion de plan de texte est notamment à penser dans son jeu avec la notion de séquence (Richer, 2004: 127):

un jeu que ne peut rendre la simple opposition global vs local dont se sert J.-M. Adam pour définir le rôle de ces deux plans d'organisation textuelle: «Les plans de textes prennent en charge l'organisation globale des textes tandis que la structuration séquentielle organise plutôt telle ou telle partie ou sous-partie (1999: 74)».

Cette dernière exigence vise à dépasser la conception additive de la totalité textuelle ainsi que celle de la séquence comme espace textuel homogène. Il faut reconnaitre que les zones textuelles où se perçoit l'homogénéité séquentielle n'apparaissent bien souvent qu'en situation scolaire où il s'agit - afin de faciliter le processus d'apprentissage - de segmenter dans le continu textuel des passages actualisant de façon typique la séquence choisie, car, même à ce niveau parcellaire du texte qu'est la séquence, l'hétérogène peut venir brouiller le délinéament de la séquence. La conception additive des séquences ne résiste pas à l'expérience ordinaire de lecture qui perçoit dans un texte des structurations surplombant la fragmentation séquentielle. L'importance des plans de textes et du niveau compositionnel est essentielle pour la mise en place d'une autre conception de la totalité textuelle. Le

10. «Les superstructures représentent pour ainsi dire une "syntaxe" globale du texte en tant que tout» (Van Dijk, I98I: 76)

11. «Plan canonique des articles de revues de psychologie, des annonces de films dans les programmes de télévision, articles de dictionnaire ou d'encyclopédies, recettes de cuisines, catalogues d'exposition, dispositio du plaidoyer de la tradition rhétorique, structures du sonnet italien ou élisabéthain, structures en actes et scènes du théâtre italien» (Adam, 1996: 33). 
niveau compositionnel qui représente une des composantes de la notion de genre ouvre alors la possibilité de théoriser les notions de segment et de séquence et ce, à travers la notion de plan de texte conventionnel.

Quelle pertinence cette notion de plan de texte ou de disposition textuelle a-t-elle pour un travail qui s'inscrit dans un cadre diachronique? Nous avons souligné à plusieurs reprises l'importance de la prise en considération de textes non narratifs pour observer des modifications au niveau des plans macro- et microstructurels.

Le discours scientifique, appartenant au type essentiellement explicatif, est un exemple particulièrement intéressant dans cette perspective. Dans sa communication présentée à l'occasion du XI ${ }^{\mathrm{e}}$ colloque international sur le moyen français (mai 2005 , Anvers), S. Bazin-Tacchella s'est intéressée à l'articulation des séquences textuelles dans la traduction française de la Chirurgia Magna de Guy de Chauliac. L'œuvre de Chauliac (I363), somme médico-chirurgicale, inventaire des connaissances héritées des Anciens et acquises par ce maître en médecine entré au service du pape Clément VI (1342-1352), a été traduite dans de très nombreuses langues vernaculaires, mais également abrégée et adaptée pour correspondre aux besoins d'un public peu lettré (les barbiers et chirurgiens barbiers). S. Bazin-Tacchella (1994: 427-438) a montré que:

La confrontation entre le texte savant, texte latin et traductions, et les textes dérivés laisse apparaître un contraste saisissant entre un discours originel, fortement articulé et ce, sur plusieurs plans, et les questionnaires ou abrégés, constitués à partir de l'œuvre de Chauliac, qui ne conservent le plus souvent de la construction initiale qu'une succession linéaire d'affirmations, parfois introduites par un simple item, au point de ressembler souvent à une sorte de catéchisme chirurgical, destiné à être appris par cœur et récité par l'apprenti-chirurgien.

La traduction française du XV $\mathrm{X}^{\mathrm{e}}$ siècle qui a été examinée par S. Bazin-Tacchella, révèle la transmission respectueuse d'un texte considéré comme un monument de la médecine scolastique. Cette «remarquable fidélité au texte latin» (Bazin-Tacchella, 2007: 62) ne concerne pas que le contenu mais également l'ordre d'exposition et le respect des plans textuels ${ }^{12}$. En effet, l'importance de la construction du savoir, la rigueur d'une construction explicite qui facilite le processus de mémorisation, est une des caractéristiques majeures du texte scolastique. Cette rigueur doit se manifester aussi bien sur le plan macrostructurel qu'au niveau microstructurel. Comme nous allons le montrer, ces deux niveaux qui constituent l'objet empirique texte, sont étroitement liés dans la mesure où la disposition textuelle - telle qu'elle est prévue par le genre - conditionne la façon dont les séquences textuelles sont enchainées et organisées (rappel des principes structurants, délimitation des séquences). L'analyse du plan macrostructurel concerne la division de l'ensemble

12. Il faut souligner l'absence de prologue dans la traduction ainsi que la rareté des gloses et le caractère anonyme de la traduction, phénomènes qui témoignent du retrait du traducteur face à l'autorité du discours originel. 
textuel en traités, doctrines ou chapitres. Le plan microstructurel est celui des propositions où il s'agit d'identifier et de décrire le fonctionnement de structurants textuels, comme, par exemple, le relatif composé lequel ou la locution quant $\grave{a}$, à laquelle sera consacrée une partie de notre étude. Selon S. Marcotte, les causes du succès de lequel en moyen français sont «d'ordre structural autant que morphologique»: «dès lors que la série qui, menacée par le démantèlement du système flexionnel, s'avérait de moins en moins efficace pour le marquage des cas, la série lequel, apte à discriminer le genre et le nombre, bénéficiait d'une nette supériorité et représentait ainsi un parti avantageux» (1997: 206). La conséquence sur le plan syntaxique est, pour la série lequel, un champ de compétence beaucoup plus étendu en moyen français qu'en français moderne. Le relatif composé joue un rôle plus fondamentalement anaphorique que syntaxique (Bazin-Tacchella, 2005: 39-40):

Deux phénomènes font apparaître l'importance de ce rôle anaphorique en moyen français, d'une part la propension de lequel à ouvrir un nouvel énoncé en le liant à ce qui précède de manière assez lâche sur le plan syntaxique [...] et d'autre part, l'emploi relativement fréquent de lequel comme adjectif relatif, dont ne subsiste en français moderne que la formule auquel cas.

Le relatif composé lequel apparaît en moyen français comme un instrument original et souple de structuration du texte scientifique descriptif.

Contrairement à ce structurant qui agit plutôt à l'intérieur d'une séquence comme outil transphrastique, la locution quant à peut servir de lien entre la macro- et la microstructure en introduisant, par exemple, une composante d'un hyperthème qui se présente au niveau de la disposition textuelle comme un titre ou sous-titre. En effet, l'établissement d'hyperthèmes et les procédés de dénombrement et de numérotation sont caractéristiques du discours scientifique. M.-C. Pouchelle a utilisé pour décrire les principes structurants appliqués par Henri de Mondeville dans sa Chirurgie ${ }^{13}$, la formule "un dénombrement obstiné des propositions» (1983: 53). Ce qui est valable pour Mondeville concerne le discours scolastique dans son intégralité. Il s'agit de parler par ordre pour faire correspondre l'ordre des choses - celles de la réalité anatomique - à celui du discours. L'ordre n'est donc pas purement formel mais il se constitue à l'image de la réalité qui règne dans l'univers chirurgical. Ainsi la cohérence du discours de l'une des traductions françaises du Compendium de epidemia (I348) - texte «officiel», rédigé par les membres de la faculté de médecine de Paris sur l'injonction du roi Philippe VI de Valois ${ }^{14}$ - est-elle assurée par deux mots-clés, Cause et Remèdes,

13. La Chirurgie est un traité de chirurgie écrit au début du XIV siècle par maittre Henri de Mondeville, chirurgien de Philippe le Bel. De I306 à I3I2 furent écrits les deux premiers traités ainsi que le premier chapitre du III traité ; l'ensemble fut traduit en I3I4.

14. Le traité est daté du mois d'octobre 1348. Il a été écrit juste avant que la capitale fût elle-même atteinte par l'épidémie de la peste. 
à partir desquels est construit le plan d'exposition du traité annoncé au point numéro 5 de l'introduction:

[6] 5. Nous entendons à faire de cest petit traictié. II. sommes. En la premiere des queles, nous enquerrons dont et comment les causes de ceste pestilunce sunt venues, sans la coignoissance des queles nul n'i puet parfaitement metre remede par medicine. En la secunde somme, nous mettrons les remedes preservatifs pour soi garder de l'epydimie et y adjoindrons aucuns pour curer.

(ms. BNF, fr. 12323; cité d'après Bazin-Tacchella, 200I : 133 ${ }^{15}$ )

Le découpage en traités, sommes et chapitres - éléments qui constituent la macrostructure d'un ensemble textuel - est une marque de l'origine scolastique du texte et met en relief les axes majeurs de la pensée médicale : causes et traitement. L'exemple [6] est extrait d'une traduction (adaptation A) qui est à dater de 1349I350. Pour A. Coville, il s'agit d'une traduction «fidèle», voire «savante» (1938: 352). $\mathrm{S}$. Bazin-Tacchella note cependant la présence de quelques différences légères mais significatives, comme, par exemple, le fait que la traduction est légèrement abrégée - ce qui implique des choix - et la présence de quelques ajouts par rapport au texte latin ${ }^{16}$.

La disposition textuelle de l'adaptation A du Compendium de epidemia répond donc à ce que le discours scientifique, autrement dit le genre explicatif issu d'un milieu universitaire, prévoit pour ce type de texte: parler par ordre, organiser des plans textuels en fonction de paramètres structurants annoncés dans la partie introductrice ou au niveau macrostructurel (titres et sous-titres). Ainsi le point numéro 5 de l'introduction à l'adaptation A du Compendium (exemple [6]) explicite-t-il la structure globale du texte. Les sixième et septième points de cette introduction insistent sur la structure complexe des deux sommes. Les composantes de l'hyperthème «.II. sommes» du point numéro 5 se transforment donc à leur tour en hyperthème dont le développement discursif comporte des termes organisateurs comme traictié et chapitre qui réapparaitront à la suite sur le plan macrostructurel dans les titres et sous-titres qui précèdent les différents fragments :

[7] 6. En la premiere somme seront .III. chapitres, pour ce que de ceste epydimie sunt .II. causes dont l'une est remote et lontaigne, car elle vient des corps celestieux de lassus, et l'autre est prochaine qui vient des corps terrestres de cha jus et se depent de la premiere de lassus, si que le premier chapitre sera de la premiere cause universele et remote, le secunt sera de la cause prochaine et particuliere, le tiers sera de pronostication et des signes, lequel sera annexé a l'un et a l'autre chapitre.

15. Pour les extraits de textes nous nous appuierons sur les traductions françaises reproduites d'après les manuscrits 12323 et 200 I par S. Bazin-Tacchella dans l'ouvrage Air, miasmes et contagion.

16. Pour une présentation détaillée de ces différences, voir S. Bazin-Tacchella (200I : IO5-I3I). 
[8] 7. De la secunde somme seront .II. traictiés, le premier sera des remedes preservatis par diete, le secunt sera des remedes preservatis et curatis par les choses medicinables. Du premier traictié seront .IV. chapitres, le premier sera de la election et rectification de l'air, le secunt de l'exercice et du baing, le tiers sera du boire et du mengier, le quart du dormir et du veillier, de inanition et repletion et des accidens de l'ame. Ou secunt traictié seront .III. chapitres, le premier sera des remedes universels, le secunt des remedes propres et particuliers, le tiers sera d'aucunes receptes qui sunt bonnes contre l'epydimie.

(Bazin-Tacchella, 200I: I33)

La progression thématique s'accomplit donc selon un principe de transformation d'une composante d'un hyperthème initialement mis en place en nouvel hyperthème. Comme nous pouvons le constater dans les exemples [6], [7] et [8], la numérotation des séquences constitue un élément explicite de construction et d'exposition du savoir. Nous sommes confrontés à l'emboîtement de deux systèmes d'ordination : d'un côté, l'énumération continue des séquences sous forme de séries appartenant aux différents plans de texte et, de l'autre, au niveau microstructurel, des séries fermées annoncées par la somme de leurs éléments («De la secunde somme seront .II. traictiés: le premier..., le secunt...»). Le dénombrement demeure l'un des procédés essentiels d'exposition du savoir, à la fois parce qu'il est un procédé de fragmentation - et donc un élément organisateur de la macrostructure - et un procédé de récapitulation.

Si nous comparons l'adaptation A du Compendium avec une autre version française (B) - celle du manuscrit fr. 200 q qui date également du milieu du XIV ${ }^{\mathrm{e}}$ siècle -, nous constatons que la macrostructure est simplifiée. En effet, le principe de transformation d'une composante d'un hyperthème en nouvel hyperthème n'est appliqué qu'une seule fois. L'adaptation B - pour laquelle le terme de traduction n'est plus valable - abandonne presque toutes les considérations sur les causes de l'épidémie, ces dernières étant simplement évoquées dans le préambule, pour ne s'intéresser qu'au régime de temps de peste. Le quatrième et dernier point du préambule est caractérisé par l'introduction de l'hyperthème trois choses qui renvoie à un ensemble de mesures à respecter pour échapper à l'épidémie. L'hyperthème est subdivisé dans le dernier énoncé de ce fragment textuel. Les trois composantes fonctionneront à leur tour comme hyperthème pour les trois points traités dans cette adaptation :

[9] 4. Combien que aucune fois pour cause de mauvese viande, l'ome encourt en ceste maladie et l'air mauvés si passe tantost au pommon et au cuer car vueilliés ou non nous attraions l'air en respirant ou la vie nous faudroit. Pourquoi il convient ceuls qui de ceste presente epydimie se voudroit garder en leur gouvernement considerer principaument trois choses. Premierement il doivent eslire meson et air convenable. Secondement il doivent vivre sobrement et de choses profitables et euls garder de nuisables. Tiercement doivent user d'aucuns remedes medicinables.

(Bazin-Tacchella, 200I: 146) 
Ce quatrième point du préambule de l'adaptation $B$ est le seul où les deux systèmes d'ordination s'emboîtent. Le flux d'information se répartit ensuite entre trois grandes sous-parties (trois points) dont chacune développe dans le rôle du thème l'une des trois composantes de l'hyperthème trois choses mis en place dans le préambule. Nous sommes donc en face d'un discours purement énumératif en trois points qui, certes, a conservé le dénombrement des séquences comme marque caractéristique du discours scolastique mais qui a réduit le phénomène complexe de l'épidémie de la peste à un seul aspect. S. Bazin-Tacchella en déduit que «la Consultation est devenue un simple Régime» (2001: III).

L'importance du dénombrement dans la perspective totalisante d'un savoir conçu comme une somme, selon la formule que M.-C. Pouchelle applique à Mondeville (1983: 52-56), se manifeste aussi dans un autre univers discursif qui se développe pendant la période du moyen français: les ouvrages didactiques. Ce qui lie le discours scientifique d'origine scolastique et le discours didactique, c'est la volonté d'expliquer des phénomènes de nature différente mais dont la complexité nécessite une présentation ou exposition particulière. En effet, comme nous allons le montrer, la construction du savoir obéit à des principes identiques. Regardons, à titre d'exemple, Le Mesnagier de Paris, écrit vers 1393 par un bourgeois de Paris, riche et vieillissant, pour sa très jeune épouse. Le terme de somme que nous avons rencontré à plusieurs reprises dans le discours scientifique, convient bien à ce type d'ouvrage dans la mesure où au Moyen Âge, tout traité didactique tend à être encyclopédique (Brereton et Ferrier (eds), 1994: 7 [introduction]):

Nous avons affaire à un enseignement comprenant des sujets aussi variés que le catéchisme, un traité de chasse à vol à l'épervier, des considérations sur les domestiques ou les maladies des chevaux; un recueil complet de recettes de cuisine côtoie toute une collection d'histoires édifiantes, puisées dans la Bible ou dans la littérature.

L'agencement rigoureux auquel obéit l'organisation du ménage selon l'idéal bourgeois peint dans le texte, se reflète aussi au niveau de la construction du texte qui - malgré ce curieux mélange des genres auquel aboutit souvent l'effort didactique - s'accomplit selon des principes structurants clairement définis dans le prologue, à la fin du quatrième point où le mari esquisse clairement les contours de son idéal féminin ${ }^{17}$ :

[ro] 4. [...] Et a la fin me semble que, se vostre affection y est telle comme vous m'avez monstré le semblant par voz bonnes paroles, il se peut acomplir en ceste maniere:

17. «4. Et toutesvoies, jasoit ce, comme j'ay dit, que a moy ne appartiengne fors que ung petit de service, si vouldroie je bien que vous sceussiez du bien et de l'onneur et de service a grant planté et foison et plus que a moy n'appartient, ou pour servir autre mary se vous l'avez aprez moy, ou pour donner plus grant doctrine a voz filles, amies ou autres, se il vous plaist et besoing en ont [...] (Brereton et Ferrier (eds), 1994: 24-26). 
c'estassavoir que une lecçon generale vous sera par moye escripte et a vous baillee sur trois distinctions contenans dixneuf articles principalement.

(Brereton et Ferrier (eds), 1994: $26^{18}$ )

La caractérisation de l'ensemble textuel comme une leçon générale répond donc à la mise en place de trois grands plans textuels qui, selon les contraintes imposées par le genre didactique, seront subdivisés en articles. Comme nous pouvons le constater, le principe de numérotation est donc également utilisé dans le genre didactique comme élément explicite de construction et comme présentation facilitant la mémorisation des leçons données par le mari. Après la mise en place de l'hyperthème des trois distinctions contenans dixneuf articles, le flux discursif s'oriente vers la présentation des différentes composantes. Ainsi les points 5,6 et 7 du prologue visent-ils la thématisation de chacune de ces trois distinctions:

[II] 5. La premiere distinction d'icelles trois est necessaire pour acquerir l'amour de Dieu [...].

(Brereton et Ferrier (eds), 1994: 26)

[I2] 6. La seconde distinction est necessaire pour le prouffit du mesnaige acroistre [...]. (Brereton et Ferrier (eds), 1994: 26)

[I3] 7. La troisiesme distinction est de jeux et esbatemens [...].

(Brereton et Ferrier (eds), I994: 26)

La répartition des dix-neuf articles entre les trois distinctions est présentée du point 8 au point 24 du prologue. Ce qui était valable pour le discours scientifique, en l'occurrence l'emboîtement de deux systèmes d'ordination, concerne donc aussi le genre didactique. Mais contrairement aux deux adaptations françaises du Compendium de epidemia, l'énumération des séquences en tant qu'outil d'organisation au niveau macrostructurel n'est plus continue, mais produit des séries fermées dont les limites permettent de séparer les éléments constitutifs de la macrostructure. Ainsi, la disposition textuelle, comme par exemple l'insertion d'un sous-titre rappelant les principes structurants avant un nouveau fragment textuel, va de pair avec l'ouverture d'une nouvelle série énumérative dont le dernier élément clôt le fragment. Cette différence concernant l'application du procédé de dénombrement s'explique, nous semble-t-il, par la complexité et la densité de l'ouvrage. En effet, à l'intérieur de chaque paragraphe il reste toujours de la place pour la digression - phénomène qui fait facilement perdre de vue l'appartenance à un sous-ensemble textuel et qui aboutit, généralement, à la composition de séquences relativement longues. Ainsi, par exemple, le sixième article de la première distinction est-il constitué de 62 paragraphes dont le huitième s'étend dans l'édition bilingue (Brereton et Ferrier (eds),

18. Tous les exemples du Mesnagier de Paris sont extraits de cette édition. 
1994) sur 20 pages $^{19}$. Le début de cet article montre que les principes structurants exposés dans le prologue ne suffisent plus pour maitriser la matière à traiter:

[I4] I. Le .VI ${ }^{\mathrm{me}}$. article de la premiere distinction dit que vous soiez humble et obeissant a cellui qui sera vostre mary, lequel article contient en soy quatre membres.

(Brereton et Ferrier (eds), 1994: 186)

L'introduction d'un nouveau principe structurant se reflète au niveau du discours par le recours à un nouvel hyperthème, en l'occurrence une série fermée annoncée par la somme de ses éléments.

Le discours scientifique et le discours didactique appartiennent au type argumentatif et explicatif. Cette caractéristique commune explique l'application de principes structurants identiques qui mettent en valeur l'importance de la construction du savoir.

Nous terminerons notre présentation centrée sur la macrostructure de textes appartenant essentiellement au type argumentatif, par quelques remarques concernant la structure globale d'un type particulier de textes car appartenant au type narratif mais se nourrissant - quant à l'organisation de la macrostructure - de principes structurants pratiqués par le genre explicatif ou argumentatif: les recueils de nouvelles (genre narratif bref). L'analyse d'un recueil comme les Cent Nouvelles Nouvelles ${ }^{20}$ $(C N N)$ soulève un problème inhérent à tout recueil de nouvelles: c'est la question de la cohésion de l'ensemble et du lien entre les différentes nouvelles. Il se peut très bien que dans un recueil il n'existe pas de lien du tout entre les différents textes qui le composent. D'un autre côté, il existe aussi des cas qui sont caractérisés par une structuration très puissante des différentes composantes. Les instruments pour l'obtention d'un tel effet sont: une action ou histoire cadre assez puissante en termes d'organisation; un inventaire limité de conteurs bien individualisés et ayant des préférences thématiques et stylistiques accusées, qui alternent dans la présentation des textes; un thème fixe pour un certain groupe de nouvelles; etc ${ }^{21}$. Entre ces deux extrêmes, toutes les positions intermédiaires sont en principe possibles. Les $C N N$ appartiennent à un groupe intermédiaire et sont à localiser plutôt du côté des recueils faiblement structurés. Au niveau de la disposition textuelle, on peut distinguer deux grands pans de texte: une introduction et le corps de texte proprement dit. L'introduction se présente sous la forme d'un inventaire commenté qui fournit un résumé pour chaque nouvelle. Le sous-titre qui est placé en tête de cet inventaire sert à introduire l'hyperthème et donc le principe structurant qui sert de fil conducteur pour la disposition textuelle et la cohésion de l'ensemble:

19. Il s'agit d'un exemple (l’histoire de Grisélidis) raconté pour mieux illustrer la thématique de cet article: «le devoir d'obéissance».

20. Le recueil anonyme des Cent Nouvelles Nouvelles fut rédigé à la demande de Philippe le Bon. Il vit le jour aux environs de I46o à la cour de Bourgogne.

21. Un cas représentatif de ce type est le Décaméron de Bocace. 
[I5] Sensuyt la table de ce present livre, intitulé des Cent Nouvelles, lequel en soy contient cent chapitres ou histoires, ou pour mieulx dire nouvelles.

(Sweetser (ed.), 1966: I)

Étant donné le titre et cette annonce faite dans la partie introductive, l'application du procédé de dénombrement s'impose donc pour l'organisation de la macrostructure du recueil. Mais contrairement au discours argumentatif et/ou explicatif, ce procédé, ici, n'aboutit pas au phénomène d'emboîtement de deux systèmes d'ordination. L'inventaire (table) annoncé dans le sous-titre ne s'appuie pas sur une numérotation en chiffres arabes, qui est un paramètre important pour l'identification du discours scolastique, mais se compose de paragraphes introduits dans tous les cas par - dans l'ordre chronologique - les composantes de l'hyperthème cent chapitres ou bistoires (La premiere nouvelle/ la secunde nouvelle/ la tierce nouvelle...). Le même principe structurant est repris dans le corps du texte où les nouvelles sont numérotées et attribuées à un conteur. En effet, il ne s'agit pas d'exposer un savoir complexe, mais d'intégrer cet ensemble de récits brefs développant des thèmes bien familiers à un lecteur médiéval ou médiéviste (satire des femmes et des maris trompés, dénonciation de la paillardise du clergé...), dans un cadre formé selon un modèle prestigieux, celui du Décaméron de Bocace, et qui implique en fonction des contraintes imposées par le genre de la nouvelle, l'application de deux grands principes narratifs: la rapidité dans le déroulement et le resserrement dans l'exposition. Que le ou les auteur(s) ai(en)t choisi de mettre en avant dans le titre le nombre de textes, a l'avantage de proposer l'image d'une ouvre qui supporte la comparaison avec le roman, puisque l'addition est signe d'abondance de matières. Chiffre rond, imposant, ce maittre mot canonique de cent va devenir une sorte de nombre d'or pour les nouvellistes.

Dans le cadre de notre étude, ce nombre d'or nous a servi à expliquer l'organisation de la macrostructure d'un recueil de nouvelles. Mais comme d'autres titres - Les Quinze Joyes de mariage (fin du XIV / début du XV $\mathrm{XV}^{\mathrm{e}}$ siècle) ou L'Heptaméron (XVI ${ }^{\mathrm{e}}$ siècle) - le font apparaitre, tout chiffre est candidat pour la mise en place d'un hyperthème qui, en tant qu'élément rassembleur, accueille un nombre bien déterminé de récits brefs qui brillent plutôt par leur technique narrative que par l'hétérogénéité des principes structurants appliqués pour organiser la macrostructure.

Indépendamment de l'appartenance d'un texte à un genre précis, ces principes structurants n'agissent pas qu'au niveau de la macrostructure. Dans la dernière partie de notre travail, nous signalerons l'importance de quelques structurants textuels pour la cohérence de l'objet empirique texte. L'analyse ne sera pas exhaustive: nous nous contenterons de quelques exemples permettant de montrer les contraintes qui s'imposent - en fonction de l'appartenance à un genre - au niveau microstructurel.

\subsection{Microstructure et structurants textuels}

Dans cette dernière partie de notre travail nous passerons de la macro- à la microstructure, c'est-à-dire au niveau des propositions. Cette partie se veut plutôt une perspective pour des recherches ultérieures consacrées au niveau microstructurel 
qu'une analyse complète de tous les outils disponibles qui assurent les liens d'une séquence à l'autre, ou à l'intérieur d'une même séquence selon le principe de continuité. En effet, la cohérence d'un discours est assurée par deux principes: celui de continuité et celui de progression. Alors que le premier principe aboutit à la diffusion dans tout le texte d'outils anaphoriques, le second principe s'exprime plus spécifiquement en début de séquence, par le choix de constructions qui ont pour fonction d'introduire un élément en position de thème, ou plus exactement de topique, si l'on suit la mise au point terminologique de B. Combettes (1999: 232).

Dans son étude consacrée aux «Organisateurs textuels et marqueurs argumentatifs en moyen français» (1986), B. Combettes soulignait l'importance dans les textes non narratifs en moyen français d'une série de locutions prépositionnelles permettant l'introduction d'un élément en position de thème, d'ordinaire à l'ouverture de la phrase; l'expression à la fois la plus fréquente et la plus représentative de cet emploi est la locution quant à. Cette locution constitue un outil linguistique capable de fonctionner au niveau de la séquence textuelle complexe (l'équivalent de notre paragraphe), comme au niveau de la phrase. Dans les deux cas, quant à fournit un cadre, indique la portée d'une prédication tout en assurant la continuité du discours par le jeu de reprises anaphoriques. L'utilisation de cet organisateur textuel concurrence donc la simple juxtaposition des idées ou même l'addition par un outil tel que item, qui est largement utilisé dans le discours scientifique et au niveau du langage didactique comme une sorte de béquille de l'argumentation.

En présentant un nouvel élément et en l'opposant le plus souvent à un ou plusieurs éléments précédents, la locution quant à fonctionne comme un organisateur textuel qui permet d'introduire les divers sous-thèmes, issus d'un même hyperthème (Combettes, 1986: 239):

Ceci est particulièrement net lorsque chaque sous-thème est développé par un passage relativement long, et non par une seule phrase; i y a là une sorte de «rappel», nécessaire, des différentes parties qui composent l'hyperthème, et l'emploi de quant $\grave{a}$ laisse en fait attendre un paragraphe.

Dans notre corpus, nous avons relevé ce type de fonctionnement surtout dans le discours savant et dans l'emploi du langage didactique. Dans l'exemple suivant, extrait de l'adaptation B du Compendium de epidemia, la locution quant à introduit la première composante de l'hyperthème trois choses (concernant le régime de temps de peste), annoncé dans le préambule:

[16] Quant au premier point, conseillent les sages et les anciens mestres que on doit de fouir les liex et les villes ou cete mortalité court et querir ayr cler et neit et le meilleur que on le pourra trouver, et qui tel ne le pourra avoir fors que gros, mauvés et corrumpu, si se tiegne en mesons basses non mie moistes, lointaignes de mauveses iaues, de charoignes et de cimetires, de courtis plains de poreaus et de chous et d'autres herbes, les quelles sont corrumpables et de toutes autres ordures et corruptions.

(Bazin-Tacchella, 200I: I46-I47). 
Ce premier sous-thème se déploie dans le fil horizontal du discours où il est repris, catégorisé et travaillé sous forme de 12 paragraphes introduits, majoritairement, par un simple et qui rattache le passage en question à la thématique abordée dans le sous-ensemble textuel. Au début de ces paragraphes, on retrouve à nouveau la locution prépositionnelle quant à qui, cette fois-ci, n'agit pas comme un structurant établissant un lien entre la macro- et la microstructure, mais thématise un référent de discours, recouvrant une réalité abstraite ou concrète, animée ou non animée. Quant à précède alors un syntagme nominal constitué d'un nom propre ou d'un nom commun (éventuellement modifiés par des expansions). Ainsi le septième paragraphe du premier point (exemple [16]) commence-t-il par «Quant aus povres, preignent une livre d'iaue rose [...]» (Bazin-Tacchella, 200I : I47), où le syntagme nominal (les) povres thématise un référent déjà introduit dans le discours sous forme d'une thématisation directe ${ }^{22}$.

Ce sont surtout les textes argumentatifs et/ou explicatifs qui fournissent de nombreux exemples pour ce type de fonctionnement assuré par des structurants textuels comme quant à, au regard de ou au propos de. B. Combettes a constaté que «ces locutions ne sont pour ainsi dire pas utilisées dans le texte narratif» (I986: 214). Dans les recueils de récits brefs de notre corpus nous n'avons relevé qu'un nombre limité d'occurrences de la locution prépositionnelle quant à. Pour les Cent Nouvelles Nouvelles, par exemple, il s'agit de sept occurrences pour lesquelles la répartition des emplois est significative pour un texte appartenant au type narratif: un emploi en tant qu'organisateur textuel avec un rôle structurant au niveau de la macrostructure (Et ce suffise quant à la première bistoire ; Sweetser (ed.), I966: 30); un emploi au niveau de la microstructure par thématisation d'un référent de discours (Et de fait elle a fait ung beau filz [...] Mais au fort, puis qu'el est telle, au dyable voit elle! Je suis bien content que le marchant l'ayt et la tienne; mais quant est de l'enfant, il est mien et si le veil ravoir; Sweetser (ed.), 1966: 148) et cinq emplois structurés selon le modèle quant à + pronom personnel (quant a moy, quant a luy, quant est de moy ${ }^{23}$ ), qui intègrent des discours directs dans lesquels ils aboutissent à un phénomène de mise en relief d'un argument/du point de vue du locuteur ou d'un autre personnage. Il est à noter que ces expressions sont placées en tête du dernier énoncé d'un discours direct, ce qui leur confère, quant au poids d'information qu'ils contiennent, un statut de contre-argument. Quant à se transforme alors en marqueur argumentatif.

Un autre type de structurant textuel que nous avons rencontré surtout dans les récits brefs de notre corpus, est constitué par la catégorie des marqueurs temporels se trouvant en tête d'énoncé. Ces adverbiaux temporels assument une double

22. Le troisième paragraphe du premier point est consacré à quelques mesures de purification de l'air à l'intention des riches. Le paragraphe suivant est introduit par: «Et pour les povres, [...]» (Bazin-Tacchella, 200I : 147).

23. Quant sert aussi de base à la variante quant est de, relativement bien représentée en moyen français. 
fonction: celle d'inauguration de séquences narratives, et celle de maintien de la cohésion entre séquences ou au sein d'une séquence.

L'extrait suivant de la huitième parmi Les Quinze Joyes de mariage - texte satirique français dont l'auteur parodie un texte de dévotion populaire, Les Quinze Joyes de la Vierge - est fortement structuré par des adverbiaux temporels qui rythment les différentes étapes d'un voyage réalisé par un mari et sa femme:

[17] Lors emprenent à aller en quelque pelerinage [...] Lors la dame dont nous parlons dit [...] Or est entreprins le veage, et se départent ensemble [...] Le bon home pense en ce veage [...] Or s'approche Quasi modo, qu'il fault partir et aller oïr les oiseaulx [...] Maintenent elle dit qu'elle a un estref trop long et l'autre trop court, maintenent luy fault son mantel, maintenant le lesse, puis dit que le cheval trote trop dur et en est malade; maintenant elle descent, et puis la fault remonter, [...], maintenant elle ne peut menger [...] Et encore les aultres femmes de la compaignie dient ainxin au bon home: [...] Or arivent au Puy en Auvergne [...] et font leurs pelerinages, [...] ; or lui baille sa femme sa sainture et ses patenoustres [...] Or y a de riches dames, damoiselles, bourgeoises, qui sont de leur compaignie [...] Or fault-il que sa femme en ait auxi bien come les autres [...].

Or s'en reviennent et autelle paine que le bon home aura eu a aller l'avra au revenir. [...] Or se rendent en la meson ou le bon home a mestier de repoux [...].

(Rychner (ed.), 1963: 66-70)

L'auteur des Quinze Joyes s'est proposé non pas de narrer des événements consécutifs, mais de démontrer une thèse: «Le mariage est une prison, une nasse». Cette image de la nasse et du poisson privé de raison encadre chaque histoire. Pour démontrer la validité de cette thèse, l'auteur se sert d'un style démonstratif. Le maintien de la cohésion au sein d'une séquence se manifeste à travers la reprise du même adverbe (or, maintenent, lors...) devant un sujet identique (Maintenent elle dit que... / maintenant (elle) le lesse/ maintenant elle descent...) ou des sujets appartenant à une même classe. Le changement d'un adverbe devant un sujet ou un type de sujet identique signale la fin d'une série d'exemples servant à illustrer la thèse ${ }^{24}$, tandis qu'un changement de sujet correspond en général à un passage de la fonction de maintien de la cohésion à celle d'inauguration de séquences narratives (exemple [17]: Or s'en reviennent $[i l s: l e$ mari et sa femme]). Le fonctionnement des marqueurs temporels est d'une certaine façon comparable au procédé de dénombrement appliqué dans le discours scientifique. Un même marqueur au début d'un énoncé signale l'appartenance à une série d'actions constituant un sous-ensemble textuel à valeur exemplaire. Un changement d'adverbial temporel va de pair avec l'introduction d'une nouvelle séquence narrative et donc d'une nouvelle série d'événements racontés pour appuyer la thèse.

24. C'est souvent le passage de lors à aucunesfois qui permet de changer de situation: «Lors la dame se tourne, et le bon homme, qui ne luy ouse desplaire, la lesse jusques au matin. Lors la dame, qui pense à son amy, et a entencion de le voir le lendemain, qui n'est pas tout un, [...] Aucunesfois avient que elle ne se lieve point, mès davant le jour el se plaint et mignote tout à escient et d'aguet» (Rychner (ed.), 1963: 54). 
4. Conclusion et perspectives

Dans le cadre de notre étude nous nous sommes intéressée au couple interactif texte/phrase dans l'histoire de la langue française et plus particulièrement pendant la période du moyen français. Deux faits justifient cette importance particulière accordée au moyen français: l'apparition de textes de type argumentatif et/ou explicatif qui témoignent de l'évolution des conceptions de la cohérence discursive, et le système de la thématisation qui se réalise d'une manière différente de celle de l'ancien français. En ce qui concerne la question de la pertinence des unités dans lesquelles se règle le fonctionnement de la cohérence textuelle, les tensions entre niveau syntaxique et niveau discursif font clairement apparaître la nécessité de relativiser des concepts comme ceux de phrase, de proposition, ou de proposition complexe. En effet, afin d'avoir accès au domaine thématique et aux conceptions de la cohérence discursive en ancien et moyen français, nous avons examiné l'interaction qui s'établit entre le niveau propositionnel (microstructure) et le plan macrostructurel en passant par le concept de genre. Comme nous l'avons montré, la disposition textuelle, la mise en place de différents plans de texte, sont liées aux genres de discours. Cette prise en considération de la notion de genre constitue, nous semble-t-il, une possibilité de dépassement d'une conception additive de la totalité textuelle. L'introduction de différents types de texte dans le corpus permet de formuler et de vérifier des hypothèses concernant le lien à établir entre le type de 
texte et les types de progressions thématiques. Nous avons constaté, par exemple, l'importance des hyperthèmes dans le discours scientifique ainsi que didactique. L'organisation de la macrostructure qui, quant à elle, se met en place selon les contraintes imposées par le genre et son historicité, a des conséquences sur le niveau propositionnel et la structuration séquentielle. Nous nous sommes attachée, dans le cadre des phénomènes de thématisation qui se manifestent sur le plan microstructurel, à présenter le fonctionnement de quelques structurants textuels permettant d'observer les relations phrase/discours et d'apprécier l'importance des types de texte. Comme nous l'avons souligné dans notre étude, l'efficacité de l'ensemble texte/discours - phrase - genre doit être éprouvée par un travail systématique genre par genre en accordant une importance particulière à la relation qui s'établit entre le plan macrostucturel et le niveau propositionnel.

\section{Bibliographie}

\section{Corpus}

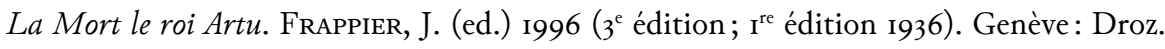

La Queste del Saint Graal. Pauphilet, A. (ed.) 2003 (3 édition; ${ }^{\mathrm{re}}$ édition 1923). Paris: Champion.

La Suite du roman de Merlin. Roussineau, G. (ed.) 2006. Genève: Droz.

Le Mesnagier de Paris. Brereton, G.E. et Ferrier, J.M. (eds) 1994. Lettres gothiques. Paris: Librairie générale française.

Les Cent Nouvelles Nouvelles. Sweetser, F.P. (ed.) 1966. Genève: Droz.

Les Quinze Joyes de mariage. Rychner, J. (ed.) 1963. Genève: Droz.

Navarre (de), M. 1950. L'Heptaméron. Paris: Garnier.

Traduction française du XV siècle de la Chirurgia Magna de Guy de Chauliac; traduction française de la Consultation de la faculté de médecine de Paris de 1348 (ms. BNF, fr. 12323); autre adaptation du Compendium (ms. BNF, fr. 20oI), à partir de:

BAZIN-TACCHELLA, S. 20OI. Rupture et continuité du discours médical à travers les écrits sur la peste de I348. Le Compendium de epidemia (1348) et ses adaptations françaises. La relation de la peste contenue dans la Chirurgia Magna de Guy de Chauliac (1363). In S. Bazin-Tacchella, D. Quéruel et É. Samana (eds), Air, miasmes et contagion: les épidémies dans l'Antiquité et au Moyen Âge. Langres: D. Guéniot: 105-156.

\section{Littérature critique}

Aрам, J.-M. 1990. Éléments de linguistique textuelle. Liège: P. Mardaga.

ADAM, J.-M. 1992. Les textes: types et prototypes. Récit, description, argumentation, explication et dialogue. Paris: Nathan.

ADAM, J.-M. 1996. L'argumentation dans le dialogue. Langue française II2: 3I-49.

ADAm, J.-M. 1997. Genres, texte, discours, pour une reconception linguistique du concept de genre. Revue belge de philologie et d'histoire 75: 3-17. 
AdAm, J.-M. 1999. Linguistique textuelle. Des genres de discours aux textes. Fac Linguistique. Paris: Nathan.

Bazin-Tacchella, S. 1994. Essai sur la diffusion et la vulgarisation de la Chirurgia Magna de Guy de Chauliac. Thèse de Doctorat. Paris IV.

Bazin-TACChella, S. 1996. Les adaptations françaises de la Chirurgia Magna de Guy de Chauliac et la codification du savoir chirurgical au XV siècle. Bien dire et bien aprandre I4: I69-I88.

BAZIN-TACCHELla, S. 2005. Le relatif lequel dans la traduction française du traité anatomique de Chauliac: un outil de cohésion. In D. James-Raoul et O. Soutet (eds), Par les mots et les textes. Mélanges de langue, de littérature et d'bistoire des sciences médiévales offerts à Claude Thomasset. Paris: Presses de l'Université de Paris-Sorbonne: 37-53.

BAZIN-TACChella, S. 2007. L'articulation des séquences textuelles dans la traduction française de la Chirurgia Magna de Guy de Chauliac: l'importance de la topicalisation. In A. VANDERHEYDEn et al. (eds), Texte et discours en moyen français. Turnhout: Brepols: $6 \mathrm{I}^{-} 72$.

Bronckart, J.-P. 1997. Activité langagière, textes et discours. Lausanne: Delachaux \& Niestlé.

BuRIDANT, C. 2000. Grammaire nouvelle de l'ancien français. Paris: SEDES.

BuridAnT, C. 2007. Connecteurs et articulation du récit en ancien et moyen français: le cas de la «Chronique des rois de France». In A. VANDERHEYDEn et al. (eds), Texte et discours en moyen français. Turnhout: Brepols: 73-94.

Cerquiglini, B., Foret, J. et Mukherjee, D. i976. Le récit saturé: l'enchainnement narratif dans la langue littéraire médiévale. Cabiers de recherche des sciences des textes et documents I: 44-64.

Combettes, B. 1983. Pour une grammaire textuelle. Bruxelles - Paris: De Boeck - Duculot.

Combettes, B. 1986. Organisateurs textuels et marqueurs argumentatifs en moyen français: «quant à». Verbum 9 (2): 213-250.

Combettes, B. 1999. Thématisation et topicalisation: leur rôle respectif dans l'évolution du français. In C. Guimier (ed.), La thématisation dans les langues. Actes du colloque de Caen (octobre 1997). Berne: Peter Lang: 23I-245.

Combettes, B. 2007. Évolution des structures thématiques en moyen français. In A. VAnderheyden et al. (eds), Texte et discours en moyen français. Turnhout: Brepols: $35-46$.

Combettes, B. et Tomassone, R. 1985. Types de progressions thématiques en moyen français. In De la plume d'oie à l'ordinateur. Numéro spécial de Verbum. Nancy: Presses universitaires de Nancy: 67-86.

Coutinho, A. 2004. Schématisation (discursive) et disposition (textuelle). In J.-M. AdAM, J.B. Grize et M. Ali Bouacha (eds), Texte et discours: catégories pour l'analyse. Dijon: Éditions universitaires de Dijon: 29-42.

Coville, A. 1938. Écrits contemporains sur la peste de 1348-I350. In Histoire littéraire de la France. Paris: Imprimerie nationale. Tome 37:352-372.

Daneš, F. 1974. Functional Sentence Perspective and the Organization of the Text. In F. Daneš (ed.), Papers on Functional Sentence Perspective. Prague: Academia: Io6-I28. 
FIRBAS, J. 197I. On the Concept of Communicative Dynamism in the Theory of Functional Sentence Perspective. In Sbornik praci filozoficke fakulty brnenske univerzity. Brno: A I9: I35-I44.

Firbas, J. 1974. Some Aspects of the Czechoslovak Approach to Problems of Functional Sentence Perspective. In F. DANEš (ed.), Papers on Functional Sentence Perspective. Prague: Academia: II-37.

Grize, J.-B. I99o. Logique et langage. Paris: Ophrys.

Hajıčova, E. 1994. Topic-Focus and Related Research. In P.A. LuELsdorfF (ed.), The Prague School of Structural and Functional Linguistics. Amsterdam - Philadelphia: John Benjamins : $245^{-276 .}$

Halliday, M.A.K. et Hasan, R. 1976. Cohesion in English. Londres: Longman.

Lehmann, S. 2007. Thématisation et organisation du discours en moyen français. In A. VANDERHEYDEn et al. (eds), Texte et discours en moyen français. Turnhout: Brepols: 47-60.

Lehmann, S. 20Io. Cohérence textuelle et progressions thématiques en moyen français: $\mathrm{XIV}^{\mathrm{e}}-\mathrm{XV} \mathrm{e}^{\mathrm{e}}$ siècles. In L. FLOREA et al. (eds), Directions actuelles en linguistique du texte. Actes du colloque international «Le texte: modèles, méthodes, perspectives». Cluj-Napoca: Editura Casa Cărții de Știință: I47-I59.

MARCotte, S. 1997. La coordination des propositions subordonnées en moyen français. Genève : Droz.

Mathesius, V. 1964. On Linguistic Characterology with Illustrations from Modern English. In J. VACHeK (ed.), A Prague School Reader in Linguistic. Bloomington : Indiana University Press : 56-63.

Pouchelle, M.-C. 1983. Corps et chirurgie à l'apogée du Moyen Âge. Paris: Flammarion.

Richer, J.-J. I997. Du type au genre: d'une approche généralisante de la littérature à une approche ouverte sur l'altérité culturelle. Études de linguistique appliquée II5: 25-37.

Richer, J.-J. 2004. Le genre: une possibilité de dépassement d'une conception additive de la totalité textuelle. In J.-M. Adam, J.B. Grize et M. Ali Bouacha (eds), Texte et discours: catégories pour l'analyse. Dijon: Éditions universitaires de Dijon: II9-I28.

Rychner, J. 1970. L'articulation des phrases narratives dans «La Mort Artu». Neuchâtel Genève : Faculté des Lettres - Droz.

Sgall, P. 1974. Zur Stellung der Thema-Rhema-Gliederung in der Sprachbeschreibung. In F. Daneš (ed.), Papers on Functional Sentence Perspective. Prague: Academia: 54-74.

VAN Dijk, T.-A. I98I. Le texte : structures et fonctions. In A. Kibedi VARGa (ed.), Théorie de la littérature. Paris: Picard.

Vennemann, T. i973a. Explanation in Syntax. In J. Kimball (ed.), Syntax and Semantics II. New York: Seminar Press : I-50.

Vennemann, T. 1973b. Language Type and Word Order. Trèves: Linguistic Agency University of Trier.

Vennemann, T. 1974a. Theoretical Word Order Studies: Results and Problems. Papiere zur Linguistik 7: 5-25. 
Vennemann, T. 1974b. Topics, Subjects and Word order: from SXV to SVX via TVX. In J.-M. Anderson et C. Jones (eds), Historical Linguistics I. Amsterdam: North-Holland Publishing Company: 339-376.

Weinrich, H. 1973. Le temps. Trad. française. Paris: Seuil ( ${ }^{\text {re }}$ édition 1964, Tempus, Stuttgart: Kohlhammer).

Wunderli, P. 1997. Le phénomène de la textualité: le texte 55 des Cent Nouvelles Nouvelles. In Le moyen français, philologie et linguistique, approches $d u$ texte et $d u$ discours. Actes $d u$ VIII colloque international sur le moyen français (Nancy, 5-7 septembre 1994). Paris: Didier Érudition : 367-39I. 